\title{
THREE-DIMENSIONAL STRUCTURE OF SOLAR WIND TURBULENCE
}

\author{
C. H. K. Chen $^{1}$, A. Mallet ${ }^{2}$, A. A. Schekochinin ${ }^{2}$, T. S. Horbury ${ }^{3}$, R. T. Wicks ${ }^{4}$, And S. D. Bale \\ ${ }^{1}$ Space Sciences Laboratory, University of California, Berkeley, CA 94720, USA; chen@ ssl.berkeley.edu \\ ${ }^{2}$ Rudolf Peierls Centre for Theoretical Physics, University of Oxford, Oxford OX1 3NP, UK \\ 3 The Blackett Laboratory, Imperial College London, London SW7 2AZ, UK \\ ${ }^{4}$ Heliophysics Science Division, NASA Goddard Space Flight Center, Greenbelt, MD 20771, USA \\ 5 Physics Department, University of California, Berkeley, CA 94720 , USA \\ Received 2012 July 5; accepted 2012 August 27; published 2012 October 8
}

\begin{abstract}
We present a measurement of the scale-dependent, three-dimensional structure of the magnetic field fluctuations in inertial range solar wind turbulence with respect to a local, physically motivated coordinate system. The Alfvénic fluctuations are three-dimensionally anisotropic, with the sense of this anisotropy varying from large to small scales. At the outer scale, the magnetic field correlations are longest in the local fluctuation direction, consistent with Alfvén waves. At the proton gyroscale, they are longest along the local mean field direction and shortest in the direction perpendicular to the local mean field and the local field fluctuation. The compressive fluctuations are highly elongated along the local mean field direction, although axially symmetric perpendicular to it. Their large anisotropy may explain why they are not heavily damped in the solar wind.
\end{abstract}

Key words: magnetic fields - magnetohydrodynamics (MHD) - plasmas - solar wind - turbulence

Online-only material: color figures

\section{INTRODUCTION}

The solar wind is a weakly collisional plasma (e.g., Kasper et al. 2008) that is ubiquitously observed to be in a turbulent state (Tu \& Marsch 1995; Goldstein et al. 1995; Horbury et al. 2005; Bruno \& Carbone 2005; Petrosyan et al. 2010; Matthaeus \& Velli 2011). Much progress has been made in understanding the nature of this turbulence since the first direct spacecraft observations (e.g., Siscoe et al. 1968; Coleman 1968) but many aspects are not yet fully understood. In particular, the threedimensional (3D) structure has been poorly characterized. Here, we use a new single-spacecraft technique to measure the $3 \mathrm{D}$ structure of turbulence in the fast solar wind.

Turbulence is usually modeled as a local cascade of fluctuations from large to small scales, forming an inertial range. In the solar wind, most of the energy at large scales is in Alfvénic fluctuations (Belcher \& Davis 1971; Bruno et al. 1985; Horbury et al. 1995; Bale et al. 2005), which have magnetic field and velocity fluctuations perpendicular to the magnetic field direction (Alfvén 1942). Early isotropic magnetohydrodynamic turbulence theories (Iroshnikov 1963; Kraichnan 1965) based on Kolmogorov scaling arguments (Kolmogorov 1941) predict that the energy spectrum of weak Alfvénic turbulence is $E(k) \sim k^{-3 / 2}$, where $k$ is the wavenumber of the fluctuations. Although one-dimensional velocity power spectra in the solar wind at 1 AU display this scaling (Mangeney et al. 2001; Podesta et al. 2007; Salem et al. 2009; Chen et al. 2011a; Boldyrev et al. 2011), the magnetic field has a $k^{-5 / 3}$ scaling (e.g., Matthaeus \& Goldstein 1982; Smith et al. 2006; Chen et al. 2011a; Boldyrev et al. 2011).

It was later realized (Montgomery \& Turner 1981; Shebalin et al. 1983) that the magnetic field direction can induce anisotropy in plasma turbulence. It was then proposed (Higdon 1984; Goldreich \& Sridhar 1995) that Alfvénic turbulence tends toward a state of critical balance, in which the timescale of the Alfvénic fluctuations propagating along the magnetic field is equal to the timescale of their nonlinear decay. This produces a spectrum perpendicular to the local magnetic field of $E\left(k_{\perp}\right) \sim k_{\perp}^{-5 / 3}$, a parallel spectrum of $E\left(k_{\|}\right) \sim k_{\|}^{-2}$ and local wavevector scaling $k_{\|} \sim k_{\perp}^{2 / 3}$. Solar wind turbulence measurements show evidence for both wavevector anisotropy of the form $k_{\perp}>k_{\|}$(Crooker et al. 1982; Bieber et al. 1996; Leamon et al. 1998; Horbury et al. 2008, 2011; Podesta 2009; Wicks et al. 2010, 2011; Chen et al. 2011b) and a steeper spectral index parallel to the local magnetic field (Horbury et al. 2008, 2011; Podesta 2009; Luo \& Wu 2010; Wicks et al. 2010, 2011; Chen et al. 2011b).

The critical balance theory was later extended to allow for the possibility that Alfvénic turbulence is 3D anisotropic (Boldyrev 2006). The two special orthogonal directions are the mean magnetic field $\mathbf{B}_{0}$ and the perpendicular magnetic field fluctuation $\delta \mathbf{B}_{\perp}$. The theory assumes that the magnetic field and velocity fluctuations align to within a scale-dependent angle $\theta_{v b}$, which makes them 3D anisotropic: $l>\xi>\lambda$, where $l, \xi$, and $\lambda$ are their correlation lengths in the mean field direction $\mathbf{B}_{0}$, in the $\delta \mathbf{B}_{\perp}$ direction, and perpendicular to both, respectively. The local spectra implied by the theory in these three directions are $E\left(k_{l}\right) \sim k_{l}^{-2}, E\left(k_{\xi}\right) \sim k_{\xi}^{-5 / 3}$, and $E\left(k_{\lambda}\right) \sim k_{\lambda}^{-3 / 2}$. The $\xi$-direction scaling follows from substituting $\xi \sim \lambda^{3 / 4}$ into $\delta v \sim \lambda^{1 / 4}$ from Boldyrev (2006), giving $\delta v \sim \xi^{1 / 3}$, corresponding to a local $-5 / 3$ spectrum.

Scale-dependent alignment has been reported in the solar wind at large scales but is difficult to measure deep in the inertial range due to instrumental limitations (Podesta et al. 2009). A recent multi-spacecraft measurement of the turbulent energy distribution in the near-Earth solar wind suggested that there was anisotropy with respect to global directions of the system, such as the global mean field, solar wind flow, or the bow shock (Narita et al. 2010b, 2010c). As far as we are aware, there has not yet been a measurement of the 3D structure of solar wind turbulence in a local, scale-dependent coordinate system $(l, \xi, \lambda)$.

Although inertial range solar wind turbulence is predominantly Alfvénic, there is also a non-negligible spectrum of compressive fluctuations $\delta B_{\|}$and $\delta n$, where $n$ is the number density 


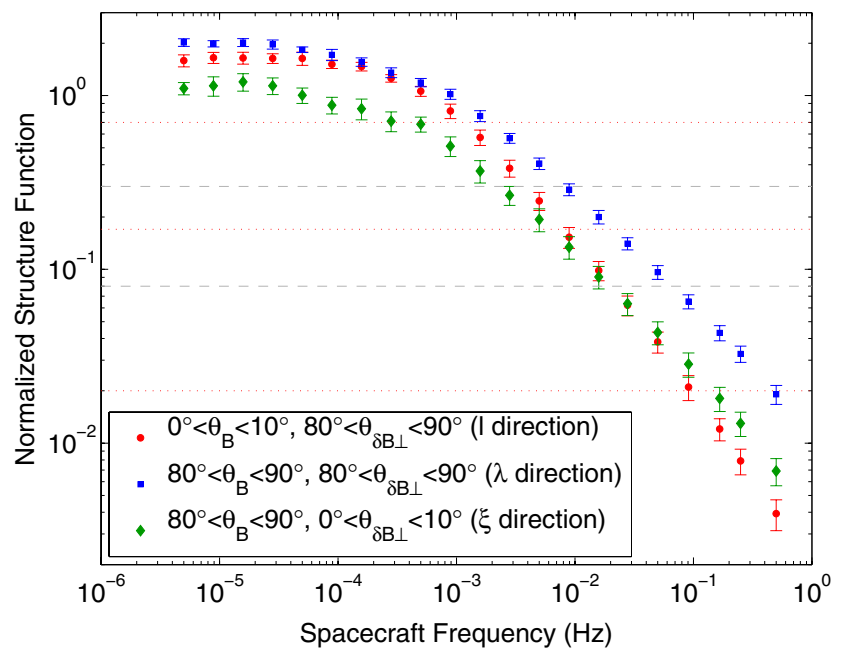

Figure 1. Normalized B-trace structure function in three orthogonal directions. The gray dashed lines indicate the range of values over which spectral indices were fitted. The red dotted lines correspond to the eddy shapes in Figure 4.

(A color version of this figure is available in the online journal.)

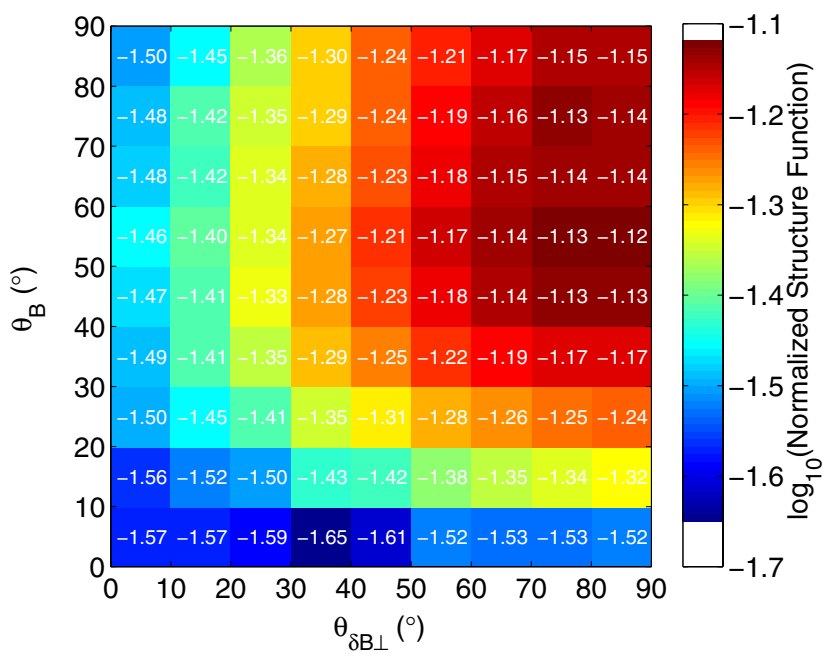

Figure 2. Normalized B-trace structure function at $1.5 \times 10^{-2} \mathrm{~Hz}$ as a function of $\theta_{B}$ and $\theta_{\delta B_{\perp}}$.

(A color version of this figure is available in the online journal.)

(e.g., Marsch \& Tu 1990; Tu \& Marsch 1994; Bavassano et al. 2004; Hnat et al. 2005; Kellogg \& Horbury 2005; Issautier et al. 2010; Chen et al. 2011a, 2012). The nature of these fluctuations is debated (Matthaeus et al. 1991; Lithwick \& Goldreich 2001; Kellogg \& Horbury 2005; Schekochihin et al. 2009; Howes et al. 2012; Klein et al. 2012), in particular, the reason why they are not heavily damped. Their structure has been less comprehensively characterized than the Alfvénic turbulence, although measurements in the magnetosheath show that there is some degree of two-dimensional (2D) anisotropy (Alexandrova et al. 2008; He et al. 2011).

In this paper, we present measurements of the scale-dependent 3D structure of the Alfvénic and compressive magnetic field fluctuations with respect to a new local coordinate system and discuss the implications for our understanding of plasma turbulence.

\section{METHOD}

In the analysis, fast solar wind data from the Ulysses spacecraft (Wenzel et al. 1992) during a polar pass between 1.4 and

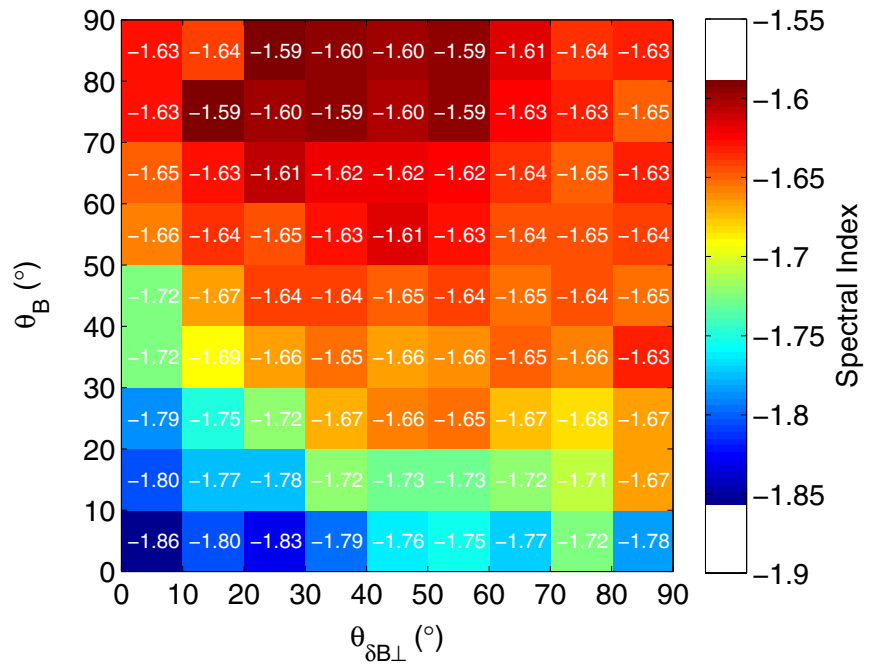

Figure 3. B-trace spectral index between normalized structure function values of 0.08 and 0.3 as a function of $\theta_{B}$ and $\theta_{\delta B_{\perp}}$.

(A color version of this figure is available in the online journal.)

2.6 AU in days 100-299 of 1995 were used. The magnetic field data from VHM (Balogh et al. 1992) were at 1 s resolution and the velocity data from SWOOPS (Bame et al. 1992) were at 4 minute resolution. The average solar wind speed was $\approx 780 \mathrm{~km} \mathrm{~s}^{-1}$ and the outer scale cross-helicity was moderately high, $\sigma_{\mathrm{c}} \approx 0.6$ (other plasma parameters for this stream are given in Wicks et al. 2010). The data were split into 10 equal length intervals for the analysis.

For each 20 day interval, 21 logarithmically spaced spacecraft-frame frequencies at which to measure the power levels, over the range $5 \times 10^{-6} \mathrm{~Hz} \leqslant f_{\mathrm{sc}} \leqslant 5 \times 10^{-1} \mathrm{~Hz}$, were chosen. For each of these frequencies, the pairs of magnetic field measurements, $\mathbf{B}_{1}$ and $\mathbf{B}_{2}$, with the time lag $1 / f_{\mathrm{sc}}$ were selected. For each pair, the contribution to the secondorder $\mathbf{B}$-trace structure function $\sum_{i}\left(B_{1, i}-B_{2, i}\right)^{2}$, where $i$ is the component of the magnetic field, and the contribution to the second-order $|\mathbf{B}|$ structure function $\left(\left|\mathbf{B}_{1}\right|-\left|\mathbf{B}_{2}\right|\right)^{2}$ were calculated. Since most of the energy is in the perpendicular fluctuations (e.g., Belcher \& Davis 1971), the B-trace spectrum is a good proxy for the Alfvénic $\delta \mathbf{B}_{\perp}$ spectrum and since $|\mathbf{B}|=\left|\mathbf{B}_{0}+\delta \mathbf{B}\right| \approx \sqrt{\left|\mathbf{B}_{0}\right|^{2}+2 \mathbf{B}_{0} \cdot \delta \mathbf{B}} \approx\left|\mathbf{B}_{0}\right|+\delta B_{\|}$, the $|\mathbf{B}|$ spectrum is a good proxy for the compressive $\delta B_{\|}$spectrum in the inertial range, where $|\delta \mathbf{B}|<\left|\mathbf{B}_{0}\right|$.

It has been shown that using a local scale-dependent $2 \mathrm{D}$ coordinate system is important for testing theoretical predictions of spectral anisotropy (Cho \& Vishniac 2000; Maron \& Goldreich 2001; Horbury et al. 2008; Chen et al. 2010a, 2011b). Here, this is extended by defining a local scale-dependent 3D coordinate system. For each pair of points, the local mean field $\mathbf{B}_{\text {local }}=\left(\mathbf{B}_{1}+\mathbf{B}_{2}\right) / 2$ and the local perpendicular fluctuation direction $\mathbf{B}_{\text {local }} \times\left[\left(\mathbf{B}_{1}-\mathbf{B}_{2}\right) \times \mathbf{B}_{\text {local }}\right]$ were calculated. The angle between $\mathbf{B}_{\text {local }}$ and the mean solar wind velocity (which is the sampling direction), $\theta_{B}$, and the angle between the local perpendicular fluctuation and the component of the solar wind velocity perpendicular to $\mathbf{B}_{\text {local }}, \theta_{\delta B_{\perp}}$, were then found.

An orthogonal spherical polar coordinate system was defined, in which $f_{\mathrm{sc}}$ is the radial coordinate, $\theta_{B}$ is the polar angle, and $\theta_{\delta B_{\perp}}$ is the azimuthal angle. It is in this local coordinate system that the 3D anisotropy of the turbulence was measured. The structure function contributions for each $f_{\mathrm{sc}}$ were binned in $10^{\circ}$ bins of $\theta_{B}$ and $\theta_{\delta B_{\perp}}$ and the mean value in each bin 

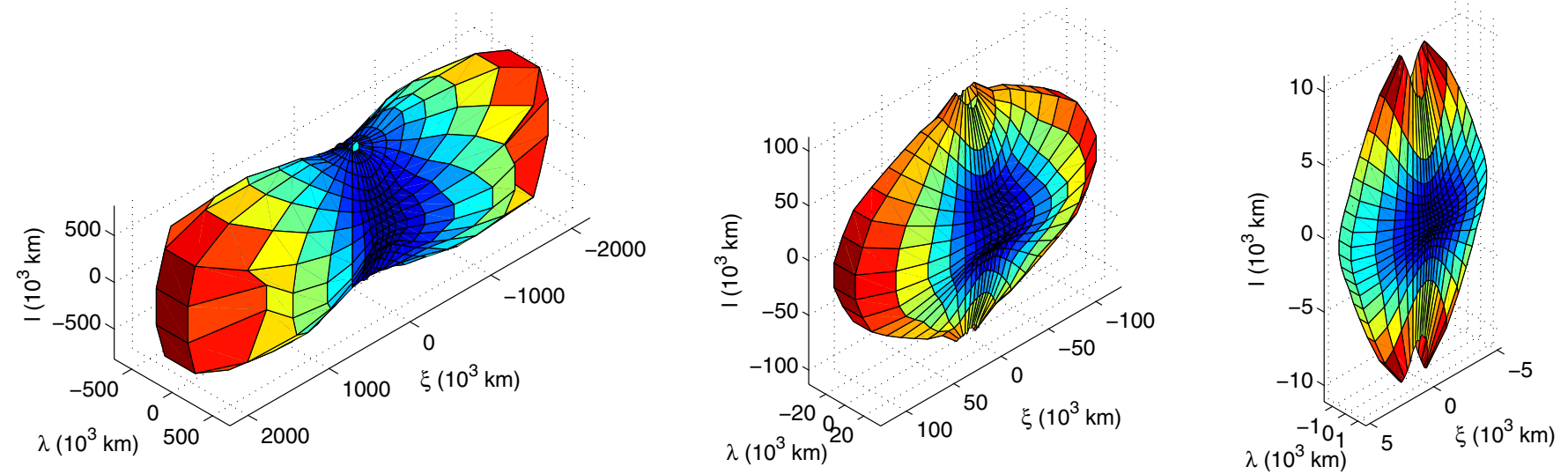

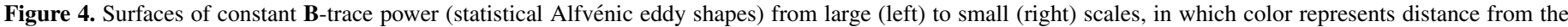
origin. The normalized power levels are $0.7,0.17$, and 0.02 as marked with red dotted lines on Figure 1 . The typical proton gyroradius is $\approx 360$ km.

(A color version of this figure is available in the online journal.)

was calculated. While the structure functions conditioned to the local field direction in this way may not be purely second order (Matthaeus et al. 2012), they are thought to be the relevant quantities in critical balance theories (Cho \& Vishniac 2000; Horbury et al. 2008; Chen et al. 2011b). Any angles greater than $90^{\circ}$ were reflected below $90^{\circ}$ to improve accuracy for scaling measurements. Reflection in $\theta_{\delta B_{\perp}}$ was found to be a good approximation; while there were few points to check the validity of reflection in $\theta_{B}$, the assumption seems reasonable (Podesta 2009).

Taylor's hypothesis (Taylor 1938) can be assumed for this analysis: since the speed of the solar wind moving past the spacecraft is more than 10 times the Alfvén speed in this interval (Wicks et al. 2010), temporal variations measured by the spacecraft, $1 / f_{\mathrm{sc}}$, correspond to spatial variations in the plasma $v_{\mathrm{sw}} / f_{\mathrm{sc}}$, where $v_{\mathrm{sw}}$ is the solar wind speed. This has been shown to be a good approximation (Narita et al. 2010a).

\section{RESULTS}

\subsection{Alfvénic Fluctuations}

Figure 1 shows the B-trace structure function ("power") as a function of spacecraft-frame frequency for three angle bins corresponding to the $\mathbf{B}_{\text {local }}$ direction (red circles), the $\delta \mathbf{B}_{\perp}$ direction (green diamonds), and the direction perpendicular to both (blue squares). Each value is the mean calculated from the 10 intervals and the error bars are $2 \sigma$, where $\sigma$ is the standard error of the mean. Before averaging, the structure functions of each interval were normalized to the square of the mean field strength over the interval $\langle|\mathbf{B}|\rangle^{2}$ to account for the varying power levels due to the spacecraft orbit. The typical proton gyroscale corresponds to a spacecraft-frame frequency $\approx 0.3 \mathrm{~Hz}$.

The perpendicular (blue) curve is characteristic of fast solar wind: shallow in the low-frequency $1 / f_{\mathrm{sc}}$ range (Matthaeus \& Goldstein 1986) and steeper in the higher frequency inertial range. The parallel (red) curve also matches previous parallel spectrum measurements, following the perpendicular curve at low frequencies, then becoming steeper than it in the inertial range (Wicks et al. 2010). The $\delta \mathbf{B}_{\perp}$ (green) curve has not previously been measured and describes how the 3D anisotropy evolves in the turbulent cascade. At large scales it has a smaller value than the other structure functions, which is consistent with this range consisting of Alfvén waves (Belcher \& Davis 1971), since they have wavevectors in the plane perpendicular to $\delta \mathbf{B}_{\perp}$.
It also remains smaller than the perpendicular structure function throughout the cascade but becomes larger than the parallel one at $\approx 3 \times 10^{-2} \mathrm{~Hz}$.

For each 20 day interval, a power law was fitted to the normalized structure functions between values of 0.08 and 0.3 (marked as gray dashed lines) in each angle bin. A fixed power range, rather than a fixed $f_{\text {sc }}$ range, was used so that the scaling was measured for the same set of fluctuations (Chen et al. $2010 b$ ). For each angle bin, the fit to the structure function was evaluated at $1.5 \times 10^{-2} \mathrm{~Hz}$ to give the $3 \mathrm{D}$ power anisotropy and the mean of the 10 intervals is shown in Figure 2. The typical standard error of the log of the mean is between 0.05 and 0.07 . It can be seen that the power increases with both $\theta_{B}$ and $\theta_{\delta B_{\perp}}$, indicating 3D anisotropy, and seems to peak near $\theta_{B}=60^{\circ}$, $\theta_{\delta B_{\perp}}=90^{\circ}$.

Each fitted power-law index was converted to a spectral index by subtracting 1 (Monin \& Yaglom 1975) and the 3D spectral index anisotropy is shown in Figure 3. The typical standard error of the mean is 0.01 or 0.02 , although the actual uncertainty may be larger due to systematic effects, such as the finite frequency response of the structure functions. The steepening toward small $\theta_{B}$ (Horbury et al. 2008) can be seen but there appears to be little variation with $\theta_{\delta B_{\perp}}$ at large $\theta_{B}$.

To visualize how the $3 \mathrm{D}$ anisotropy varies through the turbulent cascade, surfaces of constant power were calculated. At a selected structure function value, the corresponding frequency in each angle bin was found through linear interpolation and the scales corresponding to these frequencies were calculated using Taylor's hypothesis. The scales, together with the angles $\theta_{B}$ and $\theta_{\delta B_{\perp}}$, were converted into Cartesian coordinates $(l, \xi, \lambda)$ and the surfaces of constant power (at structure function values marked by red dotted lines in Figure 1) are shown in Figure 4. They have been reflected into the other seven octants under the assumption of reflectional symmetry (see earlier). These statistical surfaces can loosely be considered as average eddy shapes (although they are not eddies in the dynamical sense). It can be seen that they change from being extended in the $\delta \mathbf{B}_{\perp}$ direction in the large-scale Alfvén wave range $(\xi>l, \lambda)$ to being $3 \mathrm{D}$ anisotropic close to the proton gyroscale $(l>\xi>\lambda)$.

\subsection{Compressive Fluctuations}

The results of a similar analysis for $|\mathbf{B}|$ are shown in Figure 5 (where the structure functions have been normalized in the same way as for the Alfvénic fluctuations in Figure 1) and 


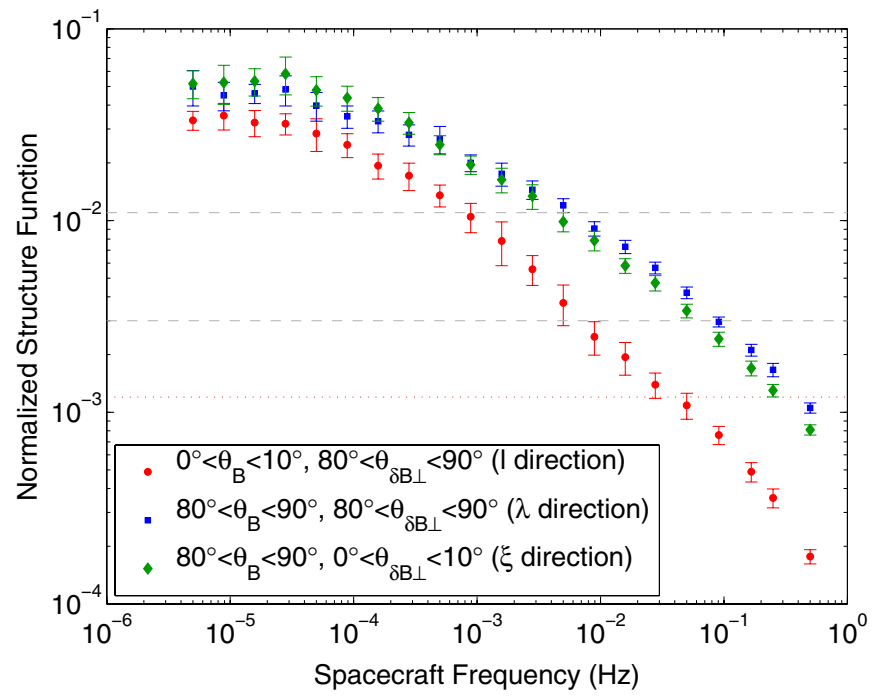

Figure 5. Normalized $|\mathbf{B}|$ structure function in three orthogonal directions. The gray dashed lines show the range of values over which spectral indices were fitted. The red dotted line corresponds to the eddy shape in Figure 6.

(A color version of this figure is available in the online journal.)

Figure 6, which is the surface of constant normalized power of $1.2 \times 10^{-3}$ (marked as a red dotted line in Figure 5). It can be seen that the structure of the compressive fluctuations is different to the Alfvénic fluctuations: there is no anisotropy in the plane perpendicular to the mean field, meaning that the compressive fluctuations do not depend on the polarization of the Alfvénic fluctuations. Also, they are more elongated along the mean field direction than the Alfvénic fluctuations: for a given perpendicular scale $\lambda$, the ratio $l / \lambda$ is at least two or three times larger. Due to limited angular resolution this is a lower limit; by extrapolating the shape in Figure 6 one could imagine that they are even more extended than can currently be measured.

The spectral indices of $|\mathbf{B}|$ for normalized powers between $3 \times 10^{-3}$ and $1.1 \times 10^{-2}$ are between -1.58 and -1.42 in all angle bins, with a typical standard error of the mean of 0.02 . This is different to the slow solar wind, where spectral indices close to $-5 / 3$ are observed (Chen et al. 2011a). This difference has also been noticed in the electron density spectrum (Issautier et al. 2010), although the reason is not well understood. If the compressive fluctuations are indeed very anisotropic, then we would not expect to measure the true parallel spectral index with the current angular resolution, which may explain the presence of anisotropic structures yet no significant anisotropic scaling.

\section{DISCUSSION}

We have shown that the Alfvénic turbulence is locally anisotropic in the plane perpendicular to the mean field. Since the direction of the anisotropy is associated with $\delta \mathbf{B}_{\perp}$, the question naturally arises to what extent this anisotropy is a reflection of the solenoidality of the magnetic field (Turner et al. 2011). While the magnetic field has zero divergence at each point, this does not imply that the correlation length along $\delta \mathbf{B}_{\perp}$ is infinite, because at any given scale we are considering finite field increments, not derivatives.

The effect of solenoidality can be derived from knowledge of the probability density function (PDF) of $\delta \mathbf{B}_{\perp}$. Since we are considering the conditional structure function at each scale $\left\langle\delta B_{\perp}^{2} \mid \theta_{\delta B_{\perp}}\right\rangle$, the joint PDF $p\left(\delta B_{\perp}, \theta_{\delta B_{\perp}}\right)$, or, equivalently, the $\mathrm{PDF}$ of the vector $\delta \mathbf{B}_{\perp}$ is required. As a simple illustration,

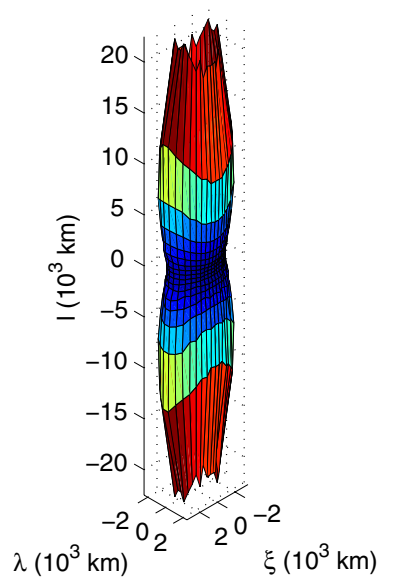

Figure 6. Surface of constant $|\mathbf{B}|$ power (statistical compressive eddy shape) at small scales. The normalized power level is $1.2 \times 10^{-3}$ as marked with a red dotted line on Figure 5.

(A color version of this figure is available in the online journal.)

consider the case where this PDF is Gaussian and, therefore, fully determined by the second-order longitudinal correlation function $C_{\mathrm{LL}}(r)=\left\langle\left|\delta \mathbf{B}_{\perp} \cdot \mathbf{r} / r\right|^{2}\right\rangle$, where $\mathbf{r}$ is the point separation in the perpendicular plane. The conditional structure function becomes

$$
\left\langle\delta B_{\perp}^{2}(r) \mid \theta_{\delta B_{\perp}}\right\rangle=\frac{2 C_{\mathrm{LL}}(r) C_{\mathrm{TT}}(r)}{C_{\mathrm{LL}}(r) \sin ^{2} \theta_{\delta B_{\perp}}+C_{\mathrm{TT}}(r) \cos ^{2} \theta_{\delta B_{\perp}}},
$$

where the transverse correlation function is $C_{\mathrm{TT}}(r)=\left[r C_{\mathrm{LL}}(r)\right]^{\prime}$ from solenoidality (Batchelor 1953). If, in the inertial range, $C_{\mathrm{LL}}(r) \propto r^{\alpha}$, then

$$
\left\langle\delta B_{\perp}^{2}(r) \mid \theta_{\delta B_{\perp}}\right\rangle \propto \frac{r^{\alpha}}{1+\alpha \cos ^{2} \theta_{\delta B_{\perp}}} .
$$

Therefore, the ratio of the correlation scales along and across the fluctuation direction for a given structure function value is scale-independent and equal to $\xi / \lambda=(1+\alpha)^{1 / \alpha}$, which, for the measured value of $\alpha \approx 2 / 3$, gives an anisotropy in the perpendicular plane of $\approx 2.15$. Since the measured anisotropy is larger than this (varying between 3.2 and 3.8), non-Gaussianity is required to explain the observations. Any scale-dependent alignment, e.g., the dynamical alignment of Boldyrev (2006), is likely to require non-Gaussianity and therefore be closely related to the intermittency of the turbulence.

Our results show some important differences to an earlier study, which suggested that the small-scale fluctuations are longest-correlated in one of the perpendicular directions and that the spectral index is different in all three directions (Narita et al. 2010b). Possible reasons for this include the different coordinate system used (global rather than local), the presence of foreshock effects in Narita et al. (2010b) or the different assumptions in the measurement technique.

The fact that the compressive fluctuations are very elongated is consistent with the prediction, based on gyrokinetic theory, that they are passive to the Alfvénic fluctuations, but have no parallel cascade along the exact magnetic field lines (Schekochihin et al. 2009). This may explain why there is a compressive cascade in the solar wind: the compressive fluctuations are expected to be damped at a rate proportional to their parallel wavenumber $\gamma \sim k_{\|}$(Barnes 1966; Schekochihin et al. 2009; Klein et al. 2012) but if $k_{\|}$is very small then they are not heavily damped 
and can cascade nonlinearly. An alternative explanation is that the less anisotropic compressive fluctuations are generated but are quickly damped, leaving the highly elongated structures to be observed.

This work was supported by NASA contract NNN06AA01C, NASA grant NNX09AE41G, and the Leverhulme Trust Network for Magnetized Plasma Turbulence. Ulysses data were obtained from CDAWeb (http://cdaweb.gsfc.nasa.gov).

\section{REFERENCES}

Alexandrova, O., Lacombe, C., \& Mangeney, A. 2008, Ann. Geophys., 26, 3585 Alfvén, H. 1942, Nature, 150, 405

Bale, S. D., Kellogg, P. J., Mozer, F. S., Horbury, T. S., \& Reme, H. 2005, Phys. Rev. Lett., 94, 215002

Balogh, A., Beek, T. J., Forsyth, R. J., et al. 1992, A\&AS, 92, 221

Bame, S. J., McComas, D. J., Barraclough, B. L., et al. 1992, A\&AS, 92, 237

Barnes, A. 1966, Phys. Fluids, 9, 1483

Batchelor, G. K. 1953, The Theory of Homogeneous Turbulence (Cambridge: Cambridge Univ. Press)

Bavassano, B., Pietropaolo, E., \& Bruno, R. 2004, Ann. Geophys., 22, 689

Belcher, J. W., \& Davis, L. 1971, J. Geophys. Res., 76, 3534

Bieber, J. W., Wanner, W., \& Matthaeus, W. H. 1996, J. Geophys. Res., 101,2511

Boldyrev, S. 2006, Phys. Rev. Lett., 96, 115002

Boldyrev, S., Perez, J. C., Borovsky, J. E., \& Podesta, J. J. 2011, ApJ, 741, L19

Bruno, R., Bavassano, B., \& Villante, U. 1985, J. Geophys. Res., 90, 4373

Bruno, R., \& Carbone, V. 2005, Living Rev. Sol. Phys., 2, 4

Chen, C. H. K., Bale, S. D., Salem, C., \& Mozer, F. S. 2011a, ApJ, 737, L41

Chen, C. H. K., Horbury, T. S., Schekochihin, A. A., et al. 2010a, Phys. Rev. Lett., 104, 255002

Chen, C. H. K., Mallet, A., Yousef, T. A., Schekochihin, A. A., \& Horbury, T. S. 2011b, MNRAS, 415, 3219

Chen, C. H. K., Salem, C. S., Bonnell, J. W., Mozer, F. S., \& Bale, S. D. 2012, Phys. Rev. Lett., 109, 035001

Chen, C. H. K., Wicks, R. T., Horbury, T. S., \& Schekochihin, A. A. 2010b, ApJ, 711, L79

Cho, J., \& Vishniac, E. T. 2000, ApJ, 539, 273

Coleman, P. J. 1968, ApJ, 153, 371

Crooker, N. U., Siscoe, G. L., Russell, C. T., \& Smith, E. J. 1982, J. Geophys. Res., 87, 2224

Goldreich, P., \& Sridhar, S. 1995, ApJ, 438, 763

Goldstein, M. L., Roberts, D. A., \& Matthaeus, W. H. 1995, ARA\&A, 33, 283

He, J., Marsch, E., Tu, C., Yao, S., \& Tian, H. 2011, ApJ, 731, 85

Higdon, J. C. 1984, ApJ, 285, 109

Hnat, B., Chapman, S. C., \& Rowlands, G. 2005, Phys. Rev. Lett., 94, 204502

Horbury, T. S., Balogh, A., Forsyth, R. J., \& Smith, E. J. 1995, Geophys. Res. Lett., 22, 3405

Horbury, T. S., Forman, M., \& Oughton, S. 2008, Phys. Rev. Lett., 101, 175005

Horbury, T. S., Forman, M. A., \& Oughton, S. 2005, Plasma Phys. Control. Fusion, 47, B703

Horbury, T. S., Wicks, R. T., \& Chen, C. H. K. 2011, Space Sci. Rev., 293

Howes, G. G., Bale, S. D., Klein, K. G., et al. 2012, ApJ, 753, L19

Iroshnikov, P. S. 1963, AZh, 40, 742
Issautier, K., Mangeney, A., \& Alexandrova, O. 2010, AIP Conf. Proc. 1216 Twelfth International Solar Wind Conference, ed. M. Maksimovic, K. Issautier, N. Meyer-Vernet, M. Moncuquet, \& F. Panteuini (Melville, NY: AIP), 148

Kasper, J. C., Lazarus, A. J., \& Gary, S. P. 2008, Phys. Rev. Lett., 101, 261103

Kellogg, P. J., \& Horbury, T. S. 2005, Ann. Geophys., 23, 3765

Klein, K. G., Howes, G. G., TenBarge, J. M., et al. 2012, ApJ, 755, 159

Kolmogorov, A. N. 1941, Dokl. Akad. Nauk SSSR, 30, 299

Kraichnan, R. H. 1965, Phys. Fluids, 8, 1385

Leamon, R. J., Smith, C. W., Ness, N. F., Matthaeus, W. H., \& Wong, H. K. 1998, J. Geophys. Res., 103, 4775

Lithwick, Y., \& Goldreich, P. 2001, ApJ, 562, 279

Luo, Q. Y., \& Wu, D. J. 2010, ApJ, 714, L138

Mangeney, A., Salem, C., Veltri, P. L., \& Cecconi, B. 2001, in Sheffield Space Plasma Meeting: Multipoint Measurements versus Theory, ed. B. Warmbein (ESA Special Publication, Vol. 492; Noordwijk: ESA), 53

Maron, J., \& Goldreich, P. 2001, ApJ, 554, 1175

Marsch, E., \& Tu, C.-Y. 1990, J. Geophys. Res., 95, 11945

Matthaeus, W. H., \& Goldstein, M. L. 1982, J. Geophys. Res., 87, 6011

Matthaeus, W. H., \& Goldstein, M. L. 1986, Phys. Rev. Lett., 57, 495

Matthaeus, W. H., Klein, L. W., Ghosh, S., \& Brown, M. R. 1991, J. Geophys, Res., 96, 5421

Matthaeus, W. H., Servidio, S., Dmitruk, P., et al. 2012, ApJ, 750, 103

Matthaeus, W. H., \& Velli, M. 2011, Space Sci. Rev., 160, 145

Monin, A. S., \& Yaglom, A. M. 1975, Statistical Fluid Mechanics, Vol. 2 (Cambridge, MA: MIT Press)

Montgomery, D., \& Turner, L. 1981, Phys. Fluids, 24, 825

Narita, Y., Glassmeier, K.-H., \& Motschmann, U. 2010a, Nonlinear Process. Geophys., 17, 383

Narita, Y., Glassmeier, K.-H., Sahraoui, F., \& Goldstein, M. L. 2010b, Phys Rev. Lett., 104, 171101

Narita, Y., Sahraoui, F., Goldstein, M. L., \& Glassmeier, K.-H. 2010c, J. Geophys. Res., 115, A04101

Petrosyan, A., Balogh, A., Goldstein, M. L., et al. 2010, Space Sci. Rev., 156, 135

Podesta, J. J. 2009, ApJ, 698, 986

Podesta, J. J., Chandran, B. D. G., Bhattacharjee, A., Roberts, D. A., \& Goldstein, M. L. 2009, J. Geophys. Res., 114, A01107

Podesta, J. J., Roberts, D. A., \& Goldstein, M. L. 2007, ApJ, 664, 543

Salem, C., Mangeney, A., Bale, S. D., \& Veltri, P. 2009, ApJ, 702, 537

Schekochihin, A. A., Cowley, S. C., Dorland, W., et al. 2009, ApJS, 182, 310

Shebalin, J. V., Matthaeus, W. H., \& Montgomery, D. 1983, J. Plasma Phys., 29,525

Siscoe, G. L., Davis, L., Jr., Coleman, P. J., Jr., Smith, E. J., \& Jones, D. E. 1968, J. Geophys. Res., 73, 61

Smith, C. W., Hamilton, K., Vasquez, B. J., \& Leamon, R. J. 2006, ApJ, 645, L85

Taylor, G. I. 1938, Proc. R. Soc. A, 164, 476

Tu, C.-Y., \& Marsch, E. 1994, J. Geophys. Res., 99, 21481

Tu, C.-Y., \& Marsch, E. 1995, Space Sci. Rev., 73, 1

Turner, A. J., Gogoberidze, G., Chapman, S. C., Hnat, B., \& Müller, W.-C. 2011, Phys. Rev. Lett., 107, 095002

Wenzel, K. P., Marsden, R. G., Page, D. E., \& Smith, E. J. 1992, A\&AS, 92, 207

Wicks, R. T., Horbury, T. S., Chen, C. H. K., \& Schekochihin, A. A. 2010, MNRAS, 407, L31

Wicks, R. T., Horbury, T. S., Chen, C. H. K., \& Schekochihin, A. A. 2011, Phys Rev. Lett., 106, 045001 